Evgeny N. Bulanov*, Maxim S. Boldin, Alexander V. Knyazev, Vitaliy Zh. Korokin and Alexander A. Popov

\title{
Obtaining Ceramic Materials from Hydroxyapatite Using Spark-Plasma Sintering
}

https://doi.org/10.1515/htmp-2016-0260

Received December 26, 2016; accepted May 4, 2017

\begin{abstract}
Hydroxyapatite was synthesized via sol-gel and solid-state reaction. Both compounds were investigated using high-temperature X-ray diffraction and differential scanning calorimetry. For sol-gel hydroxyapatite, we observed a phase transition, confirmed by character of changing of unit-cell parameters, thermal expansion coefficients and endothermic effect on DTA curve. In the process of obtaining ceramics using spark-plasma sintering both compounds manifested an unexpected shrinkage rate. All effects were explained on the basis of changings in crystal structure during distortion polymorphic transition.
\end{abstract}

Keywords: hydroxyapatite, thermal expansion, ceramics, spark-plasma sintering, high-temperature $\mathrm{x}$-ray diffraction

\section{Introduction}

Hydroxyapatite $\mathrm{Ca}_{5}\left(\mathrm{PO}_{4}\right)_{3} \mathrm{OH}$ is one of the best known inorganic compounds due to the fact that it is the main mineral component of human and animal bones $[1,2]$. Over the past 25 years, interest in the study of this substance has substantially increased: the total amount of publications on hydroxyapatite in 1985 was about 60, in 2015 - more than 3500. It can be explained by the following: today more than $50 \%$ of the population have different diseases related to bones and teeth. That is why many chemists, medics, material scientists try to obtain new materials based on hydroxyapatite and improve their properties [2].

Hydroxyapatite is commonly used as material for bone tissue engineering because it has one of the highest biocompatibility, as well as outstanding osteoinductive and osteoconductive properties [3]. It can be used in form

*Corresponding author: Evgeny N. Bulanov, Lobachevsky University, 23 Gagarina Ave., Nizhni Novgorod 603950, Russia,

E-mail: bulanoven@yandex.ru

Maxim S. Boldin, Alexander V. Knyazev, Vitaliy Zh. Korokin, Alexander A. Popov of coatings [4], ceramics [5], certain dispersions [6], etc. Another option is obtaining hydroxyapatite whiskers one-dimensional ideal crystals [7]. Such materials could be used as a reinforcing biocompatible component of composite bioceramics.

In the field of synthesis of hydroxyapatite, there are many different approaches: solution methods [8], hydrothermal approaches [9], mechano-activated ones [10]. Different methods allow to obtain hydroxyapatite with different degree of defectiveness and particle sizes, which is very important for different applications. A common problem is the inability to predict the physical, chemical and mechanical properties of hydroxyapatite obtained by different approaches. That is why every synthesized sample of hydroxyapatite should be characterized.

Obtaining hydroxyapatite ceramics using sparkplasma sintering (SPS method) is a new branch of the investigation of $\mathrm{Ca}_{5}\left(\mathrm{PO}_{4}\right)_{3} \mathrm{OH}$. The main reason for using SPS is an ability to control many parameters of the ceramics. This fact allows obtaining ceramic samples with desired mechanical properties (grain size, crack resistance, density) appropriate for different applications of the material (dentistry, surgery, etc.) [11]. Another reason is that the SPS method is the only one, which allows to obtain transparent biocompatible bioceramics for experiments with living cells and visual observation of biological processes [12].

Here we present the results of thermal investigation of hydroxyapatite, synthesized using different approaches and attempts of obtaining ceramics by spark-plasma sintering technology

\section{Experiment}

Synthesis of hydroxyapatite particles is conducted using modified sol-gel method. Initially aqueous solution of calcium nitrate is mixed with appropriate amount of $1 \mathrm{M}$ solution of phosphoric acid to achieve molar ratio $\mathrm{Ca} /$ $\mathrm{P}=5 / 3$, which is equal to stoichiometric hydroxyapatite. The process is carried out at $37^{\circ} \mathrm{C}$. At such stage the $\mathrm{pH}$ is about 1.5. In such conditions, hydroxyapatite is unstable, 
so $\mathrm{pH}$ of the solution should be increased at least to 5.5-6.0. Only after this adjustment, the precipitation of amorphous substance starts. To achieve neutral value of $\mathrm{pH}$, we used a $1 \mathrm{M}$ solution of sodium hydroxide.

At first, the precipitate consists of calcium orthophosphate. After reaching $\mathrm{pH}=6-7$ it starts to "transmutate" into hydroxyapatite. The final step of the process is isothermal holding of the mixture at $37^{\circ} \mathrm{C}$ without any stirring for $1 \mathrm{~h}$.

The obtained sol was washed using distilled water three times and centrifuged. The final substance is water dispersion of hydroxyapatite particles in water (about 5-7 wt.\%).

Synthetic process can be described using the following chemical reaction:

$5 \mathrm{Ca}\left(\mathrm{NO}_{3}\right)_{2} \cdot 4 \mathrm{H}_{2} \mathrm{O}+3 \mathrm{H}_{3} \mathrm{PO}_{4}+10 \mathrm{NaOH} \rightarrow \mathrm{Ca}_{5}\left(\mathrm{PO}_{4}\right)$ ${ }_{3} \mathrm{OH}+10 \mathrm{NaNO}_{3}+29 \mathrm{H}_{2} \mathrm{O}$

Another approach for obtaining hydroxyapatite is the traditional solid-state method [13]. The mixture of calcium hydroxyapatite, ammonium dihydrophosphate and calcium nitrate with molar ratio $\mathrm{Ca} / \mathrm{P} / \mathrm{OH}=5 / 3 / 1$ was calcined at $600^{\circ} \mathrm{C}$ for $6 \mathrm{~h}$.

For phase analysis, X-ray diffractometer XRD-6000 (Shimadzu) was used. Experiments were conducted in the $2 \theta$ range from $10^{\circ}$ to $60^{\circ}$ with scan increment of $0.02^{\circ}$ (CuK $\alpha$ radiation, geometry $\theta-2 \theta$ ).

High-temperature X-ray diffraction experiments in the range from 25 to $900^{\circ} \mathrm{C}$ were carried out on the same diffractometer with increments of $0.02^{\circ}$ ranging from $10^{\circ}$ to $60^{\circ}$ using an HA-1001 Shimadzu attachment.

Differential scanning calorimeter Labsys Setaram was used for determining of transition temperatures.

The calculating of thermal expansion coefficients was carried out using DTC program complex; plotting of thermal expansion diagram was done using KTPv2.0 software [14].

Atomic scanning microimages of hydroxyapatite particles were obtained using AURIGA CrossBeam Workstation (Carl Zeiss).

Obtaining ceramic samples was carried out at "DR. SINTER model SPS-625 Spark Plasma Sintering System” equipment (SPS Syntex Inc., Ltd., Japan).

The essence of SPS technology is a high speed (up to $2500{ }^{\circ} \mathrm{C} / \mathrm{min}$ ) heating of the material by passing a large current millisecond pulse sequence $(5000 \mathrm{~A})$ through the mold to the sintered powder material in vacuum or inert atmosphere with the simultaneous application of pressure. The attachments for precision dilatometry in modern SPS equipment used for "online" measuring of the value of time and the shrinkage rate of the powder, as well as automated systems of temperature and direct pressure control of the sintering process, enhanced this method with unique opportunities for management of structure parameters in the nano-, micro- and meso-levels. Before sintering, powders are placed in a mold, which is heated by passing current pulses through it. The duration of the pulse and delay times between them are the adjustable parameters of the system. Shrinkage of samples was monitored using precision dilatometer included in the installation kit, which allows for "on-line" mode in continuous heating and/or isothermal holding for accurate measuring of the size and velocity of the powder shrinkage. The temperature is measured by a pyrometer, focused on the outside of the mold.

Scheme of sintering regimes is represented in Figure 1. At the first stage, the rate of heating was about $200^{\circ} \mathrm{C} / \mathrm{min}$, after reaching $600^{\circ} \mathrm{C}$ it was changed to $100^{\circ} \mathrm{C} / \mathrm{min}$ (stage 2). At stage 3 , we use five temperatures $900,950,1000,1050$ and $1100^{\circ} \mathrm{C}$. At the end of the process, the cooling was carried out at $100{ }^{\circ} \mathrm{C} / \mathrm{min}$ rate. During the sintering process the pressure was $50 \mathrm{MPa}$.

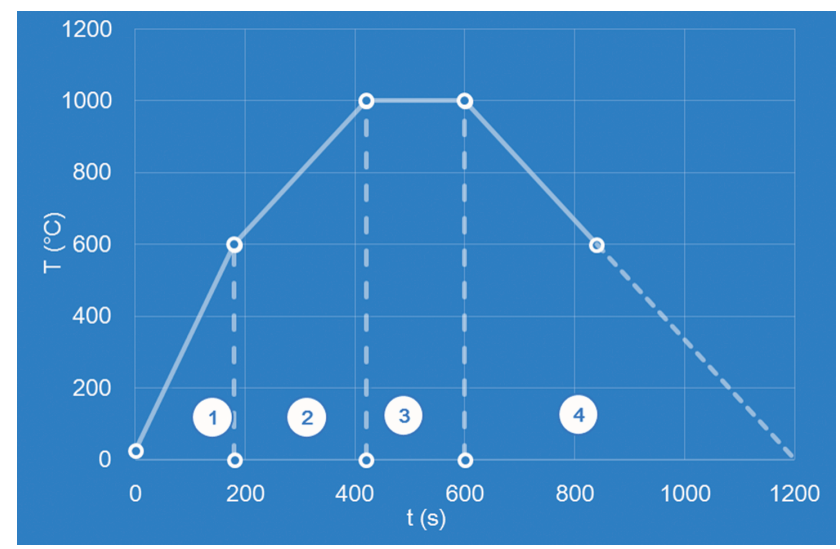

Figure 1: Scheme of sintering regimes: 1 - heating $200{ }^{\circ} \mathrm{C} / \mathrm{min}, 2-$ heating $100 \mathrm{~K} / \mathrm{min}, 3-$ isothermal holding, $4-$ cooling $100^{\circ} \mathrm{C} / \mathrm{min}$ and free cooling after $600^{\circ} \mathrm{C}$.

\section{Results and discussion}

As it follows from the XRD pattern of hydroxyapatite, synthesized via sol-gel approach (Figure 2 (curve 1)), the substance consists of nanoparticles. The corresponding calculations were made using the Scherrer equation:

$$
d=\frac{K \lambda}{\beta \cos \theta},
$$

where 


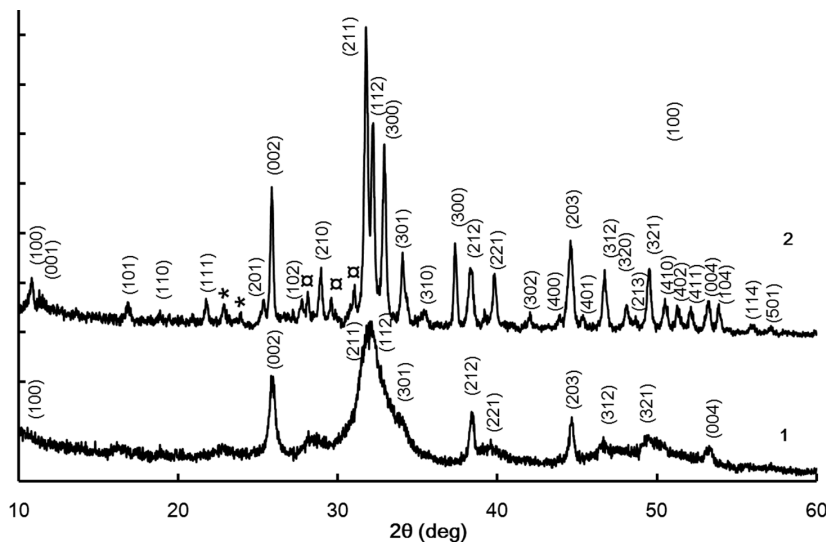

Figure 2: XRD patterns for hydroxyapatite obtained by sol-gel method (1) and solid-state reaction (2). * - cuvette reflections, a - $\beta$-tricalcium phosphate reflections.

- $d$ is the mean size of the ordered (crystalline) domains, which may be smaller or equal to the grain size;

- $K$ is a dimensionless shape factor, with a value close to unity. The shape factor has a typical value of about 0.9 , but varies with the actual shape of the crystallite;

- $\lambda$ is the $\mathrm{X}$-ray wavelength;

- $\quad \beta$ is the line broadening at half the maximum intensity (FWHM), after subtracting the instrumental line broadening, in radians. This quantity is also sometimes denoted as $\Delta(2 \theta)$;

- $\theta$ is the Bragg angle (in degrees).

Such indirect method usually renders satisfactory calculation result. In our investigation an average particle size was $47 \mathrm{~nm}$. Using scanning electron microscopy we have discovered that the "real" average particle size is about 25 nm (Figure 3).

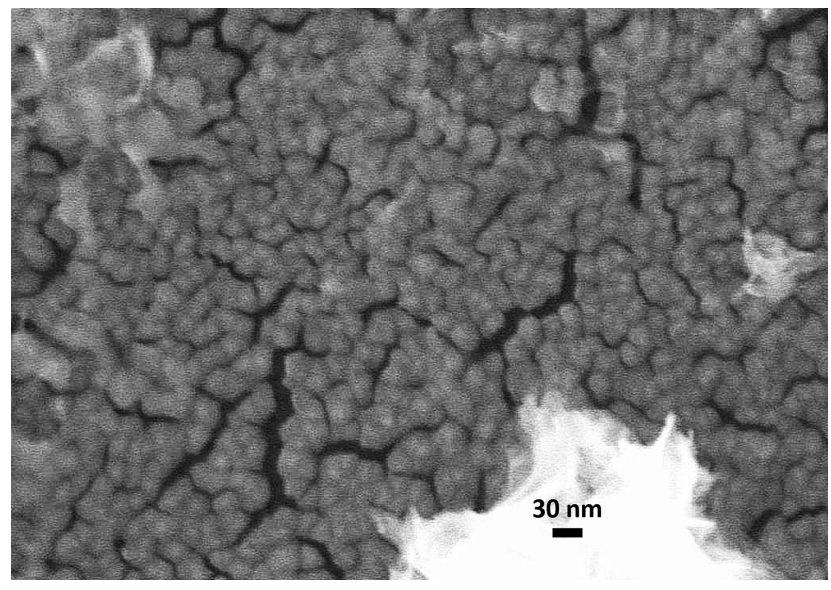

Figure 3: ASM image and XRD pattern of nanohydroxyapatite.
XRD pattern of hydroxyapatite synthesized via solidstate reaction represented in Figure 2 (curve 2).

Before making ceramic samples, obtained compounds were investigated in the range of temperature $25-1300^{\circ} \mathrm{C}$.

High-temperature investigation of both hydroxyapatites shows that their behavior at increasing temperature differs greatly. Firstly, it should be noted that they have different character of temperature dependencies of unitcell parameters. In Figure 4, temperature dependencies of unit-cell volume are represented. Moreover, it is obvious that in the case of nanohydroxyapatite, there is some changing in crystal structure because of the observed unit-cell parameters leap. In case of hydroxyapatite, synthesized using solid-state method parameter changes monotonically. Obtained data were confirmed by DSC experiment: endothermic effect at $719^{\circ} \mathrm{C}$ was observed for first hydroxyapatite, while the second one didn't have any effects (Figure 5).

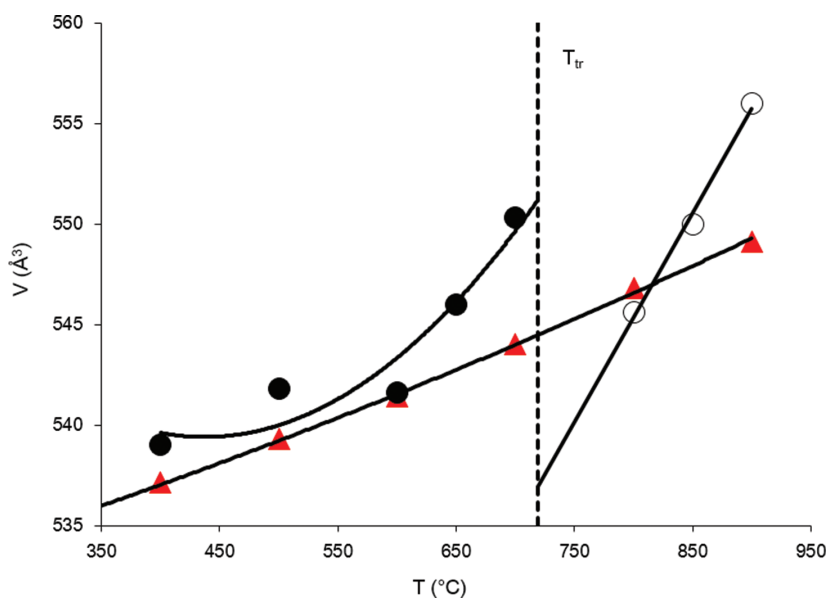

Figure 4: Thermal dependencies of unit cell volume for sol-gel hydroxyapatite before (black circles), after polymorphic transition (white circles), and for solid-state hydroxyapatite (red triangles).

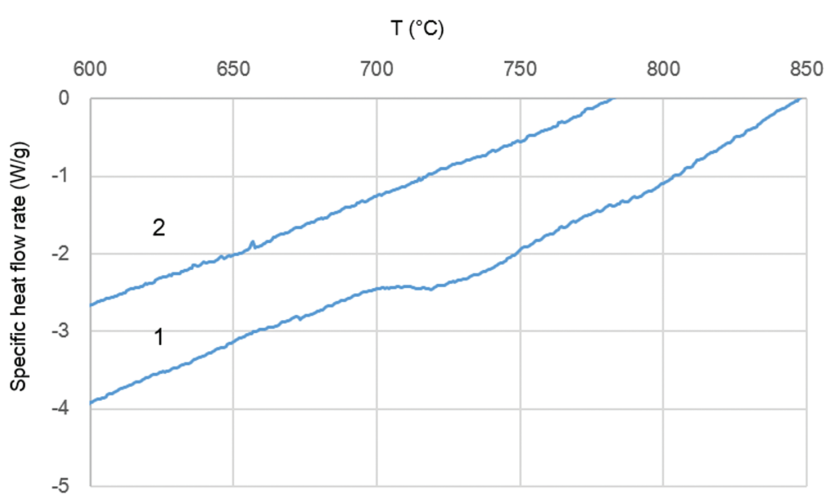

Figure 5: DTA curves for hydroxyapatite obtained by sol-gel method (1) and solid-state reaction (2). 
In addition, high-temperature X-ray diffraction method allows to calculate thermal expansion coefficients - the quantitative characteristic of thermal deformation (thermal expansion). The thermal expansion coefficient relates the change in unit-cell parameter $a$ to a change in temperature.

$\alpha_{\mathrm{a}}\left(\mathrm{K}^{-1}\right)=(1 / a)(\mathrm{d} a / \mathrm{d} T)$

In Figure 6, thermal expansion diagrams are represented. Thermal expansion diagram is the way of representing thermal expansion coefficients of the structure in different crystallographic directions. The value of the radius vector plotted from the zero-point to the boundary of the figure equals to the value of the thermal expansion coefficient in proper crystallographic direction [14].

Hydroxyapatites obtained by different methods have very different thermal expansion coefficients: nanohydroxyapatite has bigger value of $\alpha_{\mathrm{c}}$ and $\alpha_{\mathrm{a}}$ in comparison with solid-state hydroxyapatite. Moreover, anisotropy parameter $\left(\alpha_{\mathrm{c}} / \alpha_{\mathrm{a}}\right)$ for this compound is also higher. In addition, that type of hydroxyapatite has an abrupt change in the character of the expansion from anisotropic to isotropic, while the other one changes the nature of extension monotonically.

Finally, at the stage of obtaining ceramics using SPS approach, we observed unexpected effects. In the course of producing ceramics, nonlinear changing of shrinkage values of samples was found (Figure 7). Such effect was observed for both types of samples in each of the five sintering modes.

On one hand, for nanohydroxyapatite, the observed effect confirms the presence of some changes in crystal

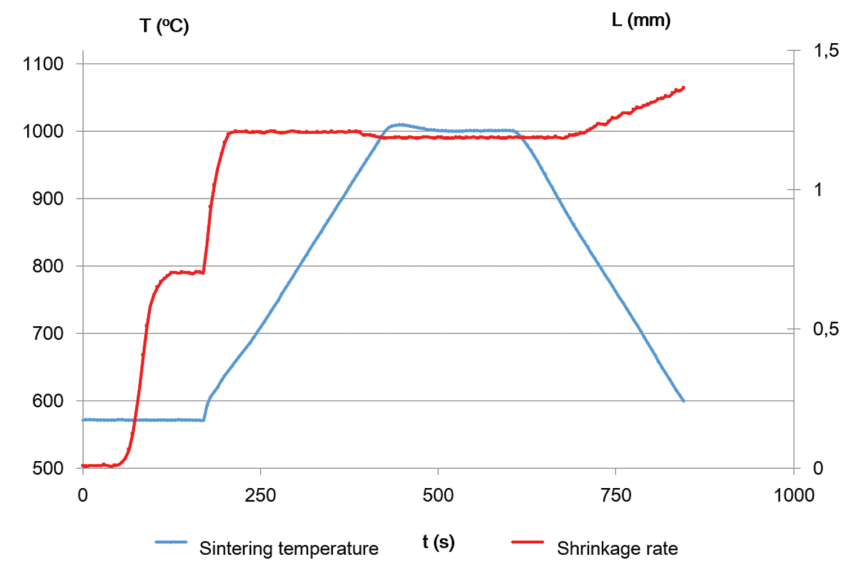

Figure 7: Typical sintering graph for hydroxyapatite (nanohydroxyapatite, temperature of isothermal holding is $1000^{\circ} \mathrm{C}$ ).

structure. On the other hand, similar effect was observed in the case of hydroxyapatite obtained by solid-state approach.

It should be noted that there are many examples of reducing of unit-cell symmetry of apatites with temperature $[15,16]$. Crystal symmetry of such compounds may change from hexagonal to monoclinic or hexagonal with another space group (e.g. $\mathrm{P}_{3} / \mathrm{m}$ to $\mathrm{P}_{3}$ ) (Figure 8). Moreover, it is well known that different structure changes in hydroxyapatite are connected with ordering/ disordering of $\mathrm{OH}$ groups. Such transitions usually have low energy (it is a distortion transition according to Burger classification [17]). That is why, in the case of solid-state synthesized hydroxyapatite we didn't determine any effects on DTA curve.

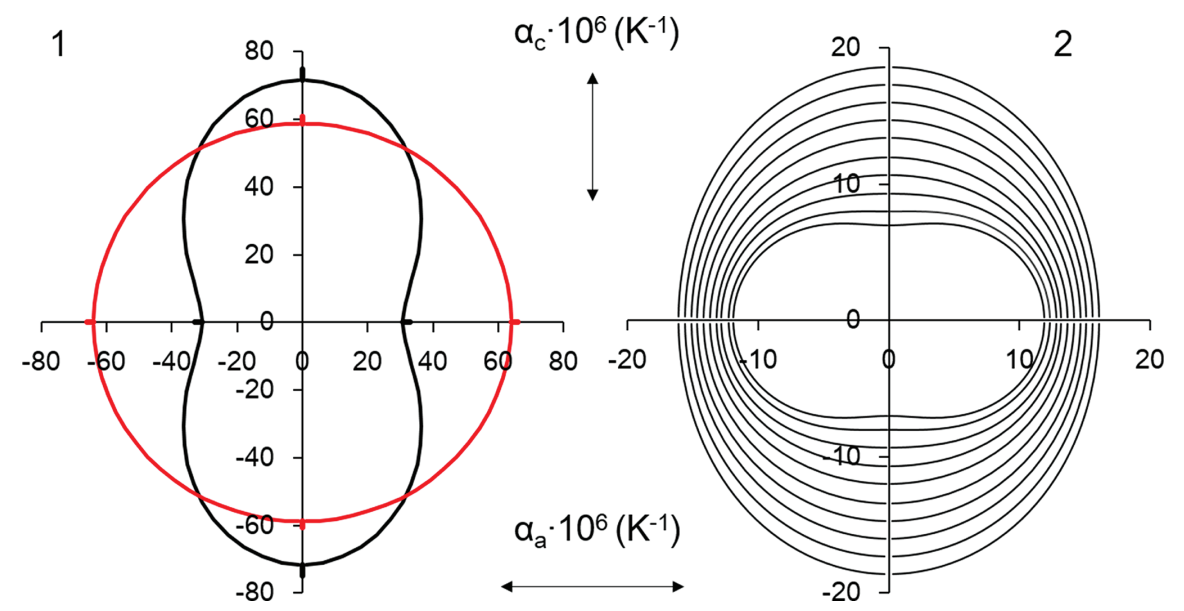

Figure 6: Thermal expansion diagrams for hydroxyapatite obtained by sol-gel method (1) (black line - before transition, red line - after transition) and solid-state reaction (2). 


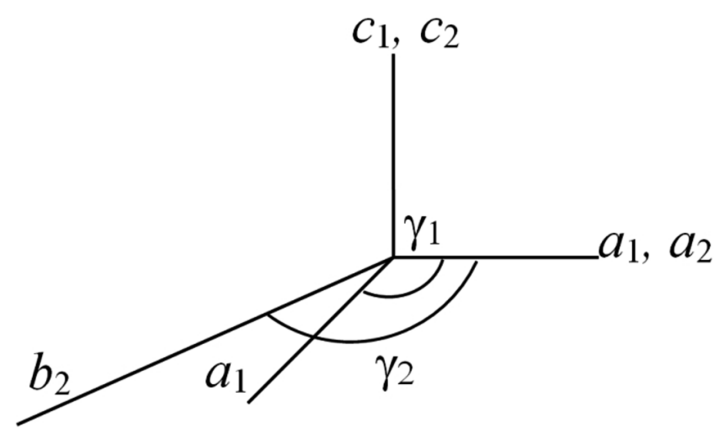

Figure 8: Scheme of changing of apatite crystal symmetry from hexagonal $(a 1, c 1, \gamma 1)$ to monoclinic $(a 2, b 2, c 2, \gamma 2)$.

\section{Conclusion}

Majority of the ceramic samples obtained by SPS approach were crashed due to mechanical stresses, which arise due to difference of thermal expansion of different polymorphic modifications of hydroxyapatite. In our opinion, there is one approach to avoid such effects. It is a chemical modification of hydroxyapatite using biocompatible ions. As it follows from literature, data compounds with apatite structure may have different character of thermal expansion. In particular, in some of them crystallographic axis a is the main direction of expansion (silicates, sulfates), while in some others it is the crystallographic axis c (phosphates, vanadates). Moreover, some apatites have negative thermal expansion, which can also be used in making chemical-modified hydroxyapatite. Using crystal-chemical modeling it is possible to create an "ideal" chemical composition, which can provide slight change of thermal expansion of a compound over the temperature range used in the SPS technology.
Acknowledgments: The work was performed with the financial support of the Russian Foundation of Basic Research (Project Number 16-33-601721 мол_а_дк).

\section{References}

1. S. Weiner and H.D. Wagner, Ann. Rev. Mater. Res., 28 (1998) 271-298.

2. S.M. Best, A.E. Porter, E.S. Thian and J. Huang, J. Eur. Ceram. Soc, 28 (2008) 1319-1327.

3. K. Yano, T. Namikawa, T. Uemura, S. Wakitani, K. Takaoka and H. Nakamura, Bone, 48(Supplement 2) (2011) S166.

4. A.M. Zhibarev, E.A. Akhmetshin and E.V. Zharikov, Russ. J. Inorg. Chem., 58(12) (2013) 1408-1411.

5. V.P. Orlovskii, V.S. Komlev and S.M. Barinov, Inorg. Mater., 38 (10) (2002) 1159-1172.

6. K.J. Lilley, U. Gbureck, J.C. Knowles, D.F. Farrar and J.E. Barralt, J. Mater. Sci.-Mater. Med., 16 (2005) 455-460.

7. A.V. Knyazev, E.N. Bulanov and E.V. Vlasova, Inorg. Mater.App. Res., 3(5) (2012) 417-420.

8. M.P. Sternlieb, H.M. Brown, C.D. Schaeffer, Jr and C.H. Yoder, Polyhedron, 28(4) (2009) 729-732.

9. Y.-P. Guo, Y.-B. Yao, Y.-J. Guo and C.-Q. Ning, Micropor. Mesop. Mat., 155 (2012) 245-251.

10. W.-L. Suchanek, P. Shuk, K. Byrappa, R.E. Riman, K.S. TenHuisen and V.F. Janas, Biomaterials, 23 (2002) 699-710.

11. Y.W. Gu, N.H. Loh, K.A. Khor, S.B. Tor and P. Cheang, Biomaterials, 23 (2002) 37-43.

12. B.-N. Kim, E. Prajatelistia, Y.-H. Han, H.-W. Son, Y. Sakka and S. Kim, Scr. Mater., 69 (2013) 366-369.

13. A.V. Knyazev, E.N. Bulanov and V.Z. Korokin, Mater. Res. Bull., 61 (2014) 47-53.

14. R.S. Bubnova and S.K. Filatov, Glass Phys. Chem., 39(3) (2013) 347-350.

15. N.G. Chernorukov, A.V. Knyazev and E.N. Bulanov, Inorg. Mater., 47(2) (2011) 172-177.

16. E.N. Bulanov and A.V. Knyazev, Chapter 7 in Apatite: Synthesis, Structural Characterization and Biomedical Applicationsedited by M. lafisco and J.M. Delgado-Lopez, Nova Science Publishers. Inc, NY (2014), pp. 173-200.

17. V.S. Urusov, Theoretical crystal-chemistry (in Russian), Moscow State University, Moscow (1987). 